\title{
openheart Two-year clinical outcomes of patients treated with the dual-therapy stent in a 1000 patient all-comers registry
}

Deborah N Kalkman, ${ }^{1}$ Pier Woudstra, ${ }^{1}$ Ian B A Menown, ${ }^{2}$ Peter den Heijer, ${ }^{3}$ Arnoud WJ van't Hof, ${ }^{4}$ Andrejs Erglis, ${ }^{5}$ Harry Suryapranata, ${ }^{6}$ Karin E Arkenbout, ${ }^{7}$ Andrés Iñiguez, ${ }^{8}$ Philippe Muller, ${ }^{9}$ Jan G Tijssen, ${ }^{1}$ Marcel A M Beijk, ${ }^{1}$ Robbert J de Winter ${ }^{1}$

\begin{abstract}
- Additional material is published online only. To view please visit the journal online (http://dx.doi.org/10.1136/ openhrt-2017-000634).
\end{abstract}

To cite: Kalkman DN, Woudstra P, Menown IBA, et al. Two-year clinical outcomes of patients treated with the dualtherapy stent in a 1000 patient all-comers registry. Open Heart 2017;4:e000634. doi:10.1136/ openhrt-2017-000634

Received 28 March 2017 Revised 7 May 2017

Accepted 13 June 2017

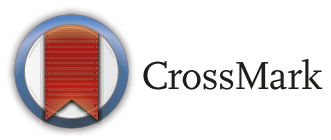

${ }^{1}$ Academic Medical Center, University of Amsterdam, Amsterdam, The Netherlands ${ }^{2}$ Craigavon Cardiac Centre, Craigavon, UK

${ }^{3}$ Amphia Hospital Breda, Breda, The Netherlands

${ }^{4}$ Isala Klinieken, Zwolle, The Netherlands

${ }^{5}$ Pauls Stradins Clinical

University Hospital, Riga, Latvia

${ }^{6}$ Radboud University Medical

Center, Nijmegen, The

Netherlands

${ }^{7}$ Tergooi Ziekenhuis, Blaricum,

The Netherlands

${ }^{8}$ Hospital Álvaro Cunqueiro

- Complejo Hospitalario

Universitario, Vigo, Spain

${ }^{9}$ Institut National de

Cardiochirurgie et de Cardiologie Interventionnelle, Luxembourg

Correspondence to Dr Deborah N Kalkman; d.n. kalkman@amc.uva.nl

\section{ABSTRACT}

Objective The dual-therapy stent combines an abluminal biodegradable drug-eluting coating, with a 'pro-healing' luminal layer. This bioengineered layer attracts circulating endothelial progenitor cells that can differentiate into normal endothelium. Rapid endothelialisation of the stent might allow safe short dual antiplatelet therapy. We aim to assess clinical outcomes in patients treated with this novel device at 2-year follow-up.

Methods A total of 1000 patients were included in the REMEDEE Registry to evaluate clinical outcomes after treatment with the dual-therapy stent. This prospective, multicentre, European registry included all-comers patients, which resulted in a high-risk patient population. Target lesion failure (TLF), a combined endpoint consisting of cardiac death, target vessel myocardial infarction (tv$\mathrm{MI}$ ) and target lesion revascularisation (TLR), at 2-year follow-up was the primary focus of this analysis. Subgroup analyses were performed according to diabetes mellitus (DM), gender, age, acute coronary syndrome, smoking, hypertension, hypercholesterolaemia, previous stroke, peripheral vascular disease and chronic renal failure. Results TLF at 2 years was observed in 84 patients (8.5\%), with $3.0 \%$ cardiac death, $1.2 \%$ tv-Ml and $5.9 \%$ TLR. Definite stent thrombosis at 2 years was $0.6 \%$. In the presence of DM or chronic renal failure, a higher TLF was observed.

Conclusions The dual-therapy stent shows favourable clinical outcomes from 12 months onwards. Two years after stent placement, low TLF and very low stent thrombosis rates are observed in this large prospective all-comers cohort study.

Trial registration number NCT01874002; Results.

\section{INTRODUCTION}

Innovations in the field of drug-eluting coronary stent (DES) therapy are rapidly evolving. First-generation DES improved outcomes in terms of reduced rates of restenosis compared with bare metal stenting. ${ }^{12}$ Subsequently, second-generation DES, with thinner stent struts, limus analogue drugs and more biocompatible polymers, were designed to reduce the increased risk of late clinical events of DES. ${ }^{3-5}$ The controversial

\section{KEY MESSAGES}

What is already known about this subject?

- Preclinical research showed early endothelialisation with this dual-therapy stent technology. One-year clinical results of the 1000 patient registry have been reported with low event rates.

What does this study add?

- There is a need for long-term clinical follow-up of novel coronary devices. This large prospective, investigator-initiated, all-comers cohort evaluates the real-world clinical performance of this dualtherapy COMBO stent at 2 years after stent placement. Low event rates are observed at 2-year follow-up.

How might this impact on clinical practice?

- The dual-therapy stent shows good 2-year clinical results and might be the preferred treatment strategy in patients with coronary artery disease, and especially in patients with indication for short dual-antiplatelet therapy. Randomised trials are needed to support this hypothesis.

term 'third-generation' DES is attributed to DES with newer (fully) biodegradable polymers and polymer-free DES. These stents and also the bioabsorbable scaffolds try to minimise adverse outcomes. ${ }^{6-8}$ However, no device has been able to eliminate in-stent neointimal hyperplasia, neoatherosclerosis and/or (very) late stent thrombosis (ST).

The COMBO stent (OrbusNeich Medical, the Netherlands) is the first dual-therapy stent (DTS), which combines the 'traditional' drug-eluting therapy (sirolimus in a biodegradable polymer) with an immobilised CD34 antibody that captures endothelial progenitor cells (EPCs) from the circulation (figure 1). These EPCs can differentiate into endothelial cells on the luminal surface of the stent. ${ }^{9}$ The hypothesis of this 'pro-healing' 


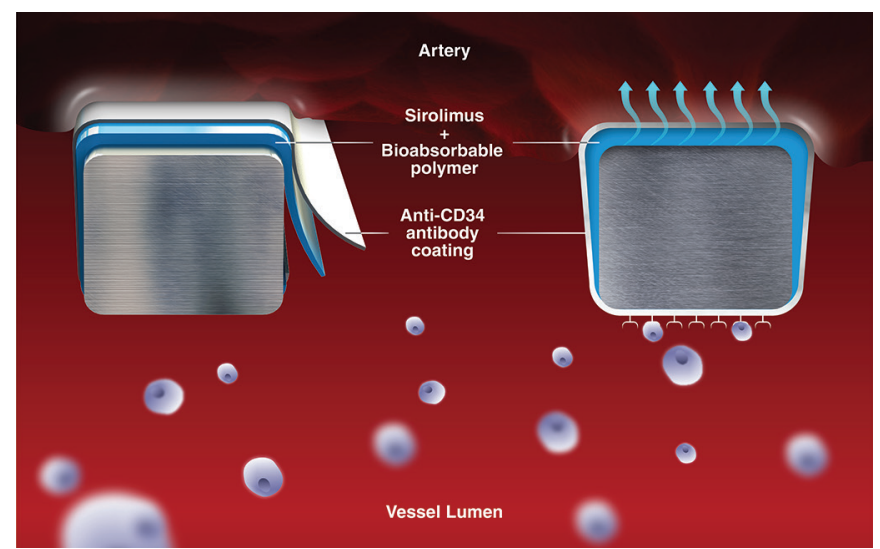

Figure 1 The COMBO stent design, illustration of the dualtherapy stent technology.

technique is that it may offer not only improved clinical outcomes, but also safe short use of dual-antiplatelet therapy (DAPT). ${ }^{1011}$

The REMEDEE Registry (all acronyms are presented in the online supplementary file 1) aims to provide more insight into the clinical outcomes with the COMBO stent in routine clinical practice and in an all-comer setting. Currently only limited long-term data are available on clinical results with the COMBO DTS exceeding 1 year. ${ }^{12}$ In this paper, we evaluate 2-year clinical results of the REMEDEE Registry and analyse outcomes in different patient subgroups.

\section{METHODS}

\section{Registry design and patient population}

The study design of the REMEDEE Registry (NCT01874002) has been previously published. ${ }^{13}$ In brief, the REMEDEE Registry is an investigator-initiated, prospective, European registry evaluating the first DTS, the COMBO stent. A total of 1000 patients, in whom treatment with a COMBO stent in the setting of routine clinical care was attempted, were enrolled. DAPT was prescribed according to local guidelines. With only few exclusion criteria, a true all-comers patient population was targeted. At 30 days, 180 days, 1-year and 2-year follow-up, all patients were contacted for clinical follow-up by telephone call or during a scheduled outpatient clinic visit. All event documentation was collected and all events were adjudicated by an independent clinical event committee.

\section{Endpoints and definitions}

In this 2-year follow-up analysis of the REMEDEE Registry, we evaluate target lesion failure (TLF), which is defined as the composite of cardiac death, target vessel myocardial infarction (tv-MI) or target lesion revascularisation (TLR). Tv-MI was defined as all myocardial infarctions (MIs) unless in the presence of documented proof that the infarction arose from a non-treated coronary vessel. TLR was defined as any repeat revascularisation by percutaneous intervention of the treated lesion or coronary artery bypass grafting (CABG) of the treated vessel. Secondary endpoints were the individual components of the primary endpoint, target vessel failure (TVF) and ST at 2-year follow-up. TVF was defined the composite of cardiac death, tv-MI and target vessel revascularisation (TVR). TVR was defined as target vessel revascularisation (any revascularisation of the treated vessel by percutaneous coronary intervention (PCI) or CABG). ST was defined according to the Academic Research Consortium criteria. $^{14}$

\section{Statistical analysis}

Categorical data are shown with counts and percentages, continuous variables are in mean $\pm \mathrm{SD}$, unless otherwise mentioned. For time-to-event data, Kaplan-Meier estimates were used for all endpoint analyses. Follow-up was censored at the last known date of follow-up, or at 24 months, whichever came first. A lost to follow-up rate of $<2 \%$ a year was deemed acceptable. All endpoints were evaluated in the unselected patient population, which consisted of all patients who were enrolled after signing an informed consent and in whom placement of a COMBO stent was attempted. Cox regression analysis was done to calculate HRs for diabetes mellitus (DM) status, gender, age, acute coronary syndrome (ACS), smoking, hypertension, hypercholesterolaemia, past stroke, peripheral vascular disease and chronic renal failure. Statistical analyses were performed using SPSS V.23.0.

\section{RESULTS}

\section{Baseline characteristics}

Between June 2013 and March 2014, 1000 patients were enrolled in nine European sites. The baseline characteristics are presented in table 1 . The mean age during baseline procedure was $65 \pm 11$ years and $26.1 \%$ of patients were female. At baseline, $58.0 \%$ of the patients had a history of hypertension and $18.4 \%$ were diabetic. The registry included $30.4 \%$ of patients with an urgent indication for PCI. TIMI flow 0 was observed in $14.5 \%$ of patients and thrombus was present in $15.0 \%$ of the cases. In two patients, the COMBO stent could not be placed. Mean stent length was $21.4 \pm 10.5 \mathrm{~mm}$ and mean diameter $3.2 \pm 0.5 \mathrm{~mm}$.

\section{Clinical outcomes}

Two-year follow-up was obtained for 981 patients $(98.1 \%)$. Events observed at 2 years after COMBO stent placement are listed in table 2. TLF occurred in 84 patients $(8.5 \%)$, as illustrated in the Kaplan-Meier curve of events found in figure 2. Cardiac death was observed in $3.0 \%$ of patients $(n=30)$, tv-MI in $1.2 \% \quad(n=12)$ and TLR in $5.9 \%(n=58)$ (figure 3). In four of the five cases of very late tv-MI, angiography was performed which did not show ST of COMBO, the fifth tv-MI was in a patient presenting with dyspnoea and decompensatio cordis, without chest pain but classified as non-ST-segment elevation MI. Echocardiography showed diffuse impaired LV function. Regional wall abnormalities could not discriminate location of MI; 


\begin{tabular}{|c|c|c|}
\hline \multicolumn{3}{|l|}{ Patients $(n=1000)$} \\
\hline Age (years) & 65 & \pm 11 \\
\hline Female & 26.1 & \\
\hline History of diabetes & 18.4 & \\
\hline Requiring insulin & 6.4 & \\
\hline History of hypertension & 58.0 & \\
\hline History of hyperlipidaemia & 56.2 & \\
\hline Family history of CAD & 45.5 & \\
\hline Current smoker & 24.1 & \\
\hline Chronic renal failure & 6.1 & \\
\hline Prior myocardial infarction & 25.3 & \\
\hline Prior percutaneous intervention & 30.1 & \\
\hline Prior $\mathrm{CABG}$ & 6.8 & \\
\hline Urgent indication for $\mathrm{PCl}$ & 30.4 & \\
\hline \multicolumn{3}{|l|}{ Lesions $(n=1255)$} \\
\hline TIMI flow 0 preprocedure & 14.5 & \\
\hline Thrombus present and thrombus aspiration & 15.0 & 10.8 \\
\hline AHA/ACC lesion type B2/C & 58.9 & \\
\hline Lesion length (mm) & 15.0 & $12-20$ \\
\hline Reference vessel diameter (mm) & 3.0 & $3.0-3.5$ \\
\hline Percentage stenosis by visual estimate & 90 & 80-99 \\
\hline Total stent length (mm) & 21.4 & \pm 10.5 \\
\hline Total stent diameter (mm) & 3.2 & \pm 0.5 \\
\hline
\end{tabular}

Values are valid $\%$, mean $\pm S D$, or median (IQR).

AHA/ACC, American Heart Association/American College of Cardiology classification; CABG, coronary artery bypass graft; $\mathrm{CAD}$, coronary artery disease; $\mathrm{PCl}$, percutaneous coronary intervention.

therefore, the CEC classified as tv-MI. Due to absence of ST-segment elevation on inferior leads (COMBO in right coronary artery), the possibility of an ST was found less likely by the CEC.

Table 2 Clinical endpoints at 1-year and 2-year follow-up

\begin{tabular}{lllll}
\hline & \multicolumn{2}{l}{$\begin{array}{l}\text { 1-year } \\
\text { up }\end{array}$} & \begin{tabular}{l} 
follow- \\
\cline { 2 - 5 }
\end{tabular} & \multicolumn{2}{c}{$\begin{array}{l}\text { Total 2-year } \\
\text { follow-up }\end{array}$} \\
\hline TLF & \% & N & $\%$ \\
\hline Cardiac death & 57 & 5.7 & 84 & 8.5 \\
\hline Target vessel MI & 17 & 1.7 & 30 & 3.0 \\
\hline TLR & 7 & 0.7 & 12 & 1.2 \\
\hline TVR & 43 & 4.3 & 58 & 5.9 \\
\hline TVF & 48 & 4.8 & 70 & 7.1 \\
\hline Definite ST & 62 & 6.2 & 96 & 9.7 \\
\hline Probable ST & 5 & 0.5 & 6 & 0.6 \\
\hline
\end{tabular}

MI, myocardial infarction; ST, stent thrombosis; TLF, target lesion failure; TLR, target lesion revascularisation; TVF, target vessel failure; TVR, target vessel revascularisation.

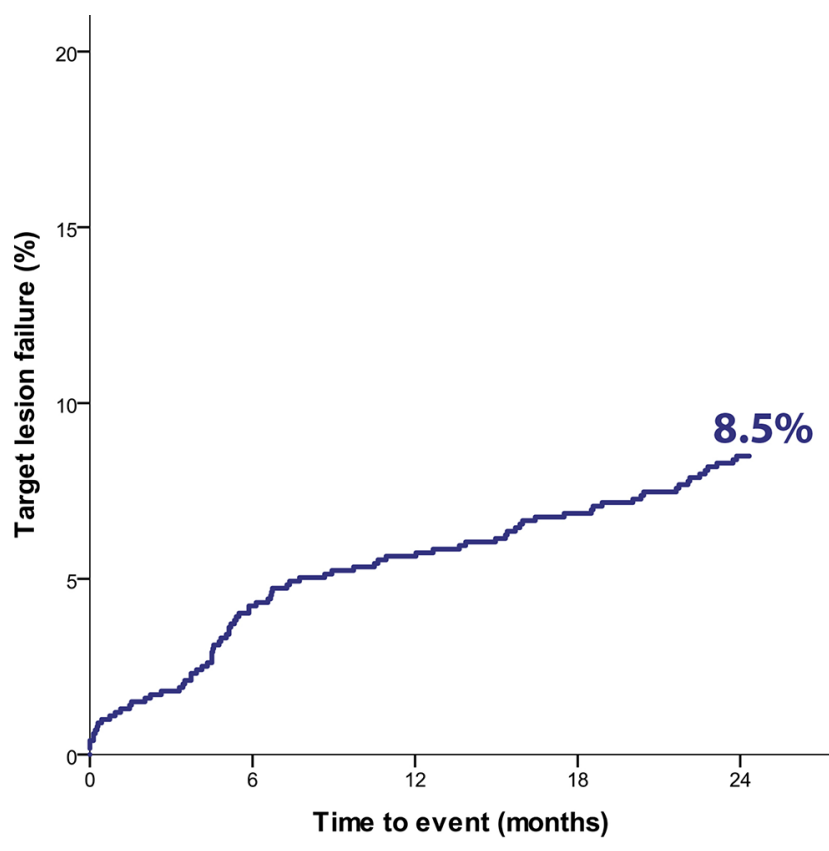

$\begin{array}{lllll}1000 & 950 & 932 & 909 & 891\end{array}$

Figure 2 Cumulative event rate of target lesion failure by Kaplan-Meier method.

A total of 70 patients underwent TVR $(7.1 \%)$, resulting in a $9.7 \%$ TVF at 2 years. Definite ST at 2 years occurred in six patients $(0.6 \%)$. Five patients had ST within the first nine days post procedure, one patient with ACS had a very late ST at 380 days, shortly after DAPT cessation.

The influence of baseline patient characteristics (gender, age, cardiovascular risk factors, ACS, peripheral vascular disease and chronic renal failure) on 2-year clinical outcome is shown in figure 4 . The only significant univariate predictors of TLF were diabetes HR $3.00(95 \%$ CI 1.93 to 4.66) and chronic renal failure HR 2.47 (95\% CI 1.31 to 4.67 ), although trends were seen with current smokers, ACS and peripheral vascular disease.

\section{DISCUSSION AND LIMITATIONS \\ Key findings}

This study reports the clinical outcomes up to 2-year follow-up in a large prospective, all-comers cohort of patients treated with the COMBO stent. We found (1) low rates of clinical events observed at 2 years after COMBO DTS implantation with an overall low percentage of the primary endpoint TLF of $8.5 \%$ and very late ST in only one patient $(0.1 \%)$, (2) our subgroup analyses found that the COMBO DTS gave favourable results in several high-risk patient groups although higher rates of TLF were observed at 2 years in patients with DM and chronic renal failure higher rates of TLF,and (3) the rate of TLR between 1 and 2 years follow-up was very low.

\section{Clinical implications}

Currently only limited data are available on clinical outcomes with the COMBO DTS exceeding 1-year follow-up. ${ }^{12}$ The results presented in this study are in line 

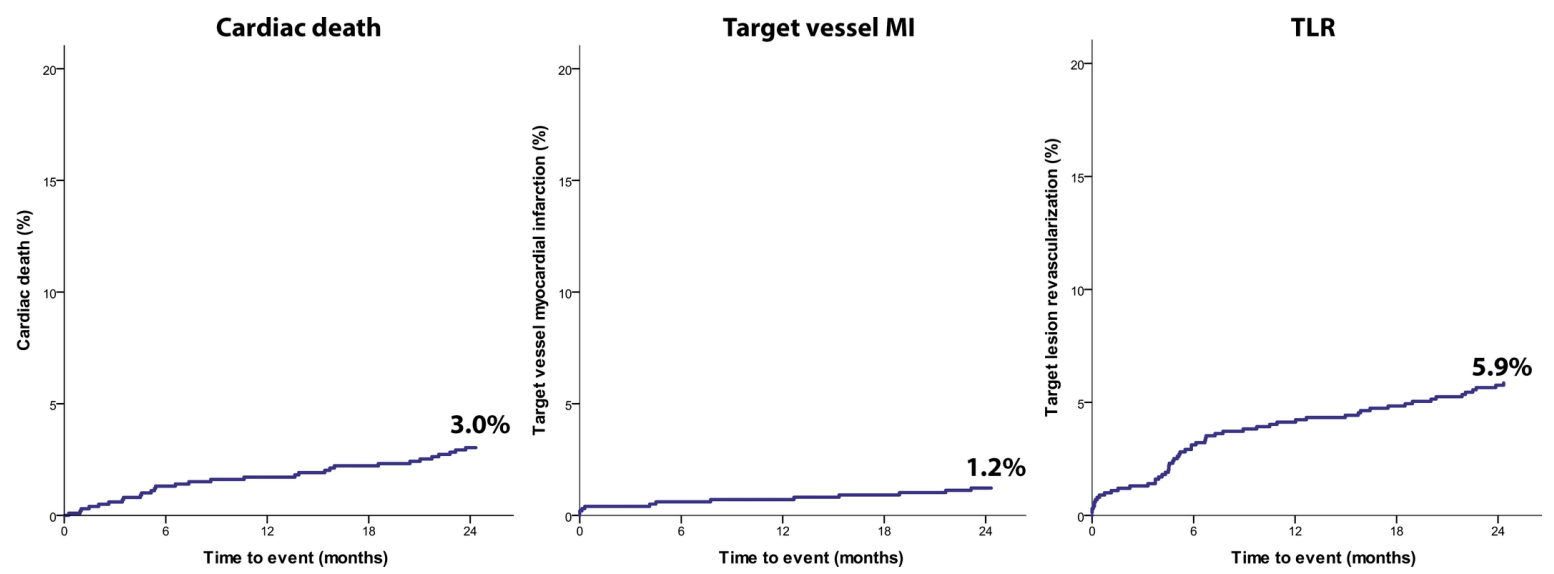

Figure 3 Cardiac death, target vessel myocardial infarction (MI), target lesion revascularisation (TLR) by Kaplan-Meier method. (A) Cumulative event rate of the individual endpoint cardiac death. (B) Cumulative event rate of the individual endpoint target vessel-related MI. (c) Cumulative event rate of the individual endpoint TLR.

with other newer-generation DES (see figure 5) such as the all-comers TWENTE ${ }^{15}$ and RESOLUTE (see online supplementary file 1$)^{16}$ trials which compared a zotarolimus-eluting stent (ZES) (RESOLUTE, Medtronic, USA) with an everolimus-eluting stent (EES) (XIENCE V, Abbott Vascular, USA). In addition, compared with the 2-year outcomes of the more selected patient population SPIRIT IV trial, comparing a paclitaxel-eluting stent (PES) (TAXUS, Boston Scientific, USA) with an EES (XIENCE, Abbott Vascular, USA), reported similar outcomes. ${ }^{17}$

ST in the REMEDEE Registry was reassuringly low despite the all-comers design. In particular, very late ST (between 12 and 24 months) occurred in only $0.1 \%$ of patients receiving the COMBO stent (which is lower than with the other devices above; EES varies 0.1-0.3, ZES $0.3 \%$ and PES $0.2 \%) .{ }^{15-17}$ A recent meta-analysis by Nairooz et al reports a 2-year ST rate of the bioresorbable vascular scaffold of $2.1 \% .^{18}$ The low ST rate at 2 years with the COMBO stent is a significant finding supporting the

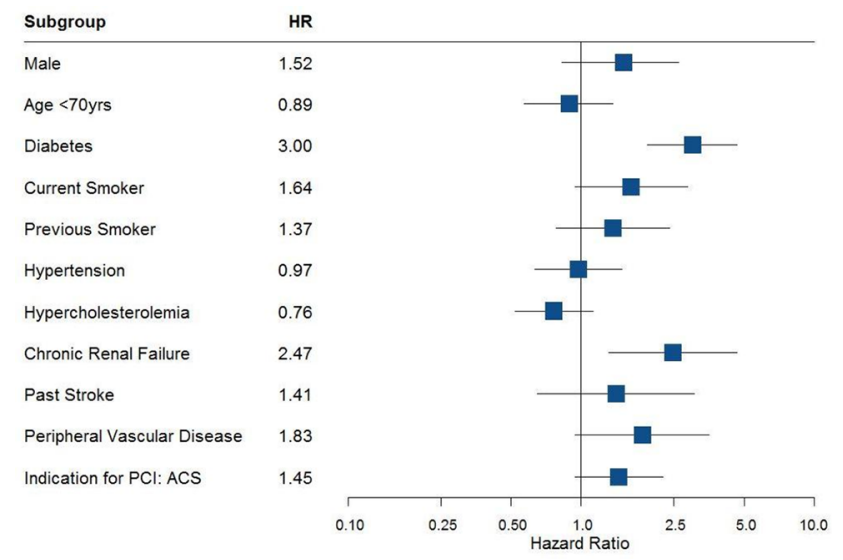

Figure 4 Subgroups. HR of gender, age, diabetic status, smoking (current and previous), hypertension, hypercholesterolaemia, chronic renal failure, previous stroke, peripheral vascular disease and acute coronary syndrome (ACS) on target lesion failure at 2-year follow-up. PCI, percutaneous coronary intervention
DTS technology hypothesis of low ST risk due to early endothelialisation of the stent, but caution should be taken and randomised data are needed to confirm these findings.

\section{Neointimal regression}

A recent study evaluating the healing pattern of the COMBO stent at 4-monthly groups (1:2:2:1 ratio from 2 to 5 months), 9 months and 24 months showed a new phenomenon not described earlier with any other DES: neointimal regression visualised by optical coherence tomography between 9 and 24 months. ${ }^{12}$ This intimal hyperplasia regression has also been assessed by quantitative coronary analyses previously in the Genous stent (OrbusNeich, a BMS with EPC capturing layer). ${ }^{19}$ These consistent findings may support the hypothesis of the beneficial effect of rapid endothelialisation and healthy maturation of the endothelial layer by means of EPC capture by the immobilised CD34 antibody at the stent surface. This study showed low TLR rate between 1 and 2 years (from $4.3 \%$ to $5.9 \%$; a $1.6 \%$ increase). Moreover, these results emphasise the need for evaluation of

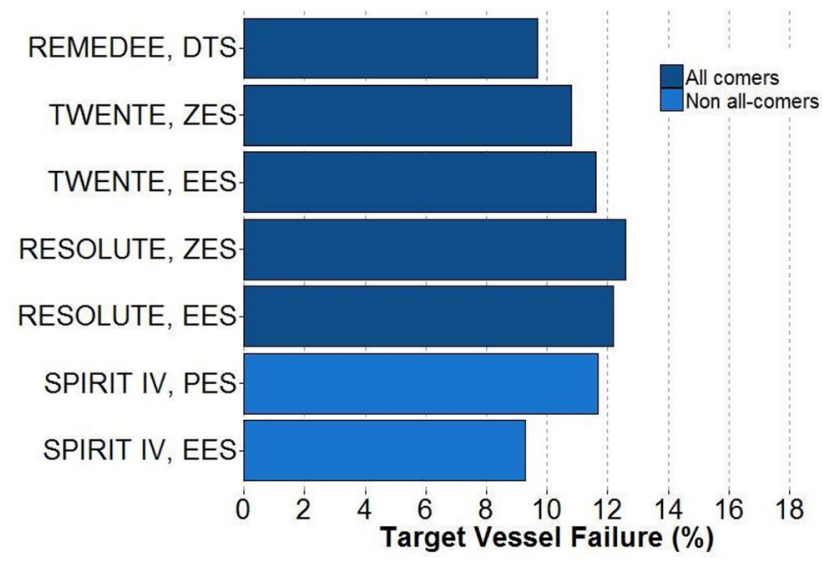

Figure 5 Comparison of target vessel failure at 2-year follow-up with other drug eluting stents (DES). DTS, dualtherapy stent; EES, everolimus-eluting stent; PES, paclitaxeleluting stent; ZES, zotarolimus-eluting stent. 
long-term clinical results, exceeding 1-year follow-up, to make the true comparison of performance of the DES.

\section{Subgroups}

DM is a known risk factor for higher adverse outcome after PCI. ${ }^{20}{ }^{21}$ We published 1-year clinical outcomes after COMBO stent placement of insulin-treated DM, non-insulin-treated DM and non-DM patients, with a clear signal of higher TLF in insulin-treated DM. ${ }^{22}$ Although patients with chronic kidney disease (CKD) often have DM, after multiple regression analyses in the DM group at 1 year, CKD was also independently associated with higher TLF. Clinical outcomes of patients with CKD and stent placement should further be explored.

\section{Future perspectives}

The REMEDEE Registry will evaluate patients up to 5 years after COMBO stent placement, and thereby, will give further insight into the long-term treatment effect of DTS technology with COMBO. Currently the US-Japan randomised HARMONEE trial (see online supplementary file 1), which evaluates clinical outcomes between EES (XIENCE family, Abbott Vascular) versus COMBO, awaits completion of 1-year follow-up. This study will also obtain clinical follow-up up to 5 years too.

\section{Limitations}

The main limitation of the REMEDEE Registry was the single-arm design. However, data collection was undertaken with great care to ensure optimum data quality. On-site monitoring and full-event documentation were collected for independent adjudication by the clinical event committee. Another limitation of the registry design is that the DAPT duration was not mandated by the registry's protocol; therefore, the majority of patients received DAPT for 1 year. A potential benefit of the COMBO DTS would be illustrated more clearly if a short duration of DAPT (eg, <6 months) after COMBO stent placement would show the same low ST rates. However, this study is the first large cohort to report on long-term results after $\mathrm{COMBO}$ stent in an all-comer patient population and provides new insight into the performance of DTS.

\section{CONCLUSION}

Two-year clinical results with the COMBO stent in the 1000 subject REMEDEE Registry have shown low rates of TLF, TVF and ST. These results confirm the long-term clinical effectiveness of DTS technology in an all-comers patient population.

Acknowledgements All patients, catheterisation laboratory nurses and interventional cardiologists are greatly acknowledged for their participation and efforts regarding the REMEDEE Registry. The authors especially acknowledge Margriet Klees for all her efforts regarding the REMEDEE Registry.

Contributors DNK and PW contributed to the conception, design, analysis and interpretation of data and drafting of the manuscript and final approval of the manuscript submitted. IBAM, PdH, AWJvH, AE, HS, KEA, Al, PM, and MAMB contributed to the conception and design of data and revising manuscript critically for important intellectual content and final approval of the manuscript submitted. JGT and RJdW contributed to the conception, design, analysis and interpretation of data and drafting of the manuscript and revising manuscript critically for important intellectual content and final approval of the manuscript submitted.

Funding The Academic Medical Center, University of Amsterdam received an unrestricted research grant from OrbusNeich Medical B.V., the Netherlands.

Competing interests None declared.

Patient consent Obtained.

Ethics approval A waiver was obtained from the ethics committee of the Academic Medical Center, University of Amsterdam.

Provenance and peer review Not commissioned; externally peer reviewed.

Data sharing statement The relevant anonymised patient-level data are available on reasonable request to the corresponding author.

Open Access This is an Open Access article distributed in accordance with the Creative Commons Attribution Non Commercial (CC BY-NC 4.0) license, which permits others to distribute, remix, adapt, build upon this work non-commercially, and license their derivative works on different terms, provided the original work is properly cited and the use is non-commercial. See: http://creativecommons.org/ licenses/by-nc/4.0/

(C) Article author(s) (or their employer(s) unless otherwise stated in the text of the article) 2017. All rights reserved. No commercial use is permitted unless otherwise expressly granted.

\section{REFERENCES}

1. Morice MC, Serruys PW, Sousa JE, et al. RAVEL Study Group. Randomized Study with the Sirolimus-Coated Bx Velocity BalloonExpandable Stent in the Treatment of Patients with de Novo Native Coronary Artery Lesions. A randomized comparison of a sirolimuseluting stent with a standard stent for coronary revascularization. $N$ Engl J Med 2002;346:1773-80.

2. Greenhalgh J, Hockenhull J, Rao N, et al. Drug-eluting stents versus bare metal stents for angina or acute coronary syndromes. Cochrane Database Syst Rev 2010:CD004587.

3. Navarese EP, Kowalewski M, Kandzari D, et al. First-generation versus second-generation drug-eluting stents in current clinical practice: updated evidence from a comprehensive meta-analysis of randomised clinical trials comprising 31379 patients. Open Heart 2014;1:e000064.

4. Park KW, Kang SH, Velders MA, et al. Safety and efficacy of everolimus- versus sirolimus-eluting stents: a systematic review and meta-analysis of 11 randomized trials. Am Heart J 2013;165:241-50.

5. Alfonzo F, Fernandez C. Second-Generation moving the field forward. J Am Coll Cardiol Elsevier Inc 2011;58:26-9.

6. Costa RA, Abizaid A, Mehran R, et al. Polymer-Free Biolimus A9Coated Stents in the treatment of De Novo coronary Lesions. JACC Cardiovasc Interv 2016;9:51-64

7. Stefanini GG, Byrne RA, Serruys PW, et al. Biodegradable polymer drug-eluting stents reduce the risk of stent thrombosis at 4 years in patients undergoing percutaneous coronary intervention: a pooled analysis of individual patient data from the ISAR-TEST 3, ISAR-TEST 4, and LEADERS randomized trials. Eur Heart J 2012;33:1214-22.

8. Indolfi C, De RS, Colombo A. Bioresorbable vascular scaffolds. Nat Rev Cardiol 2016. Epub.

9. Klomp M, Beijk MAM, de Winter RJ. Genous ${ }^{\mathrm{TM}}$ endothelial progenitor cell-capturing stent system: a novel stent technology. expert rev Med Devices. Taylor \& Francis 2009;6:365-75.

10. Larsen K, Cheng C, Tempel D, et al. Capture of circulatory endothelial progenitor cells and accelerated re-endothelialization of a bio-engineered stent in human ex vivo shunt and rabbit denudation model. Eur Heart J 2012;33:120-8.

11. Camaro C, Damen SA, Brouwer MA, et al. Randomized evaluation of short-term dual antiplatelet therapy in patients with acute coronary syndrome treated with the COMBO dual therapy stent: rationale and design of the REDUCE trial. Am Heart J 2016;178:37-44.

12. Swl L, Scc L, Fcc T, et al. Evaluation of early healing Profile and Neointimal transformation over 24 months using longitudinal sequential Optical Coherence Tomography assessments and 3-Year clinical results of the New Dual-Therapy endothelial progenitor cell capturing Sirolimus-Elu. Circ Cardiovasc Interv 2016.

13. Woudstra P, Kalkman DN, den Heijer P, et al. 1-Year results of the REMEDEE?Registry. JACC Cardiovasc Interv 2016;9:1127-34 http:// linkinghub.elsevier.com/retrieve/pii/S193687981630142X. 
14. Cutlip DE, Windecker S, Mehran R, et al. Clinical end points in coronary stent trials: a case for standardized definitions. Circulation 2007;115:2344-51 http://circ.ahajournals.org/cgi/doi/.

15. Tandjung K, Sen H, Lam MK, et al. Clinical outcome following stringent discontinuation of dual antiplatelet therapy after 12 months in real-world patients treated with second-generation zotarolimus-eluting resolute and everolimus-eluting Xience $\mathrm{V}$ stents: 2-year follow-up of the randomized TWENTE trial. J Am Coll Cardiol 2013;61:2406-16.

16. Silber $S$, Windecker $S$, Vranckx $P$, et al. Unrestricted randomised use of two new generation drug-eluting coronary stents: 2-year patient-related versus stent-related outcomes from the RESOLUTE All Comers trial. Lancet 2011;377:1241-7.

17. Stone GW, Rizvi A, Sudhir K, et al. Randomized comparison of everolimus- and Paclitaxel-Eluting Stents: 2-year Follow-Up from the SPIRIT (Clinical evaluation of the XIENCE V Everolimus Eluting Coronary Stent System) IV trial. J Am Coll Cardiol. Elsevier Inc 2011;58:19-25.
18. Nairooz R, Saad M, Sardar P, et al. Two-year outcomes of bioresorbable vascular scaffold versus drug-eluting stents in coronary artery disease: a meta-analysis. Heart 2017.

19. Klomp M, Beijk MA, Tijssen JG, et al. Significant intimal hyperplasia regression between 6 and 18 months following Genous endothelial progenitor cell capturing stent placement. Int J Cardiol 2011:147:289-91.

20. Fr?bert O, Lagerqvist B, Carlsson J?rg, et al. Differences in Restenosis Rate with Different Drug-Eluting Stents in Patients with and without Diabetes Mellitus. J Am Coll Cardiol 2009;53:1660-7.

21. Jensen LO, Thayssen $P$, Junker $A$, et al. Comparison of outcomes in patients with versus without diabetes mellitus after revascularization with everolimus- and sirolimus-eluting stents (from the SORT OUT IV trial). Am J Cardiol 2012;110:1585-91.

22. Kalkman DN, Woudstra P, den Heijer P, et al. One year clinical outcomes in patients with insulin-treated diabetes mellitus and non-insulin-treated diabetes mellitus compared to non-diabetics after deployment of the bio-engineered COMBO stent. Int J Cardiol 2017;226:60-4. 